\title{
An explicit variance reduction expression for the Rao-Blackwellised particle filter
}

Fredrik Lindsten, Thomas B. Schön, Jimmy Olsson

Division of Automatic Control

E-mail: lindsten@isy.liu.se, schon@isy.liu.se, jimmy@maths.1th.se

20th October 2010

Report no.: LiTH-ISY-R-2979

Submitted to 18th World Congress of the International Federation of Automatic Control (IFAC)

Address:

Department of Electrical Engineering

Linköpings universitet

SE-581 83 Linköping, Sweden

WWW: http://www.control.isy.liu.se

AUTOMATIC CONTROL

REGLERTEKNIK LINKÖPINGS UNIVERSITET

Technical reports from the Automatic Control group in Linköping are available from http://www . control.isy.liu.se/publications. 


\begin{abstract}
Particle filters (PFs) have shown to be very potent tools for state estimation in nonlinear and/or non-Gaussian state-space models. For certain models, containing a conditionally tractable substructure (typically conditionally linear Gaussian or with finite support), it is possible to exploit this structure in order to obtain more accurate estimates. This has become known as Rao-Blackwellised particle filtering (RBPF). However, since the RBPF is typically more computationally demanding than the standard PF per particle, it is not always beneficial to resort to Rao-Blackwellisation. For the same computational effort, a standard PF with an increased number of particles, which would also increase the accuracy, could be used instead. In this paper, we have analysed the asymptotic variance of the RBPF and provide an explicit expression for the obtained variance reduction. This expression could be used to make an efficient discrimination of when to apply Rao-Blackwellisation, and when not to.
\end{abstract}

Keywords: Particle filtering, Monte-Carlo methods, Rao-Blackwellised particle filter, Marginalised particle filter, Rao-Blackwellisation, variance reduction 


\title{
An explicit variance reduction expression for the Rao-Blackwellised particle filter
}

\author{
Fredrik Lindsten* Thomas B. Schön* Jimmy Olsson** \\ * Division of Automatic Control, Linköping University, Linköping, \\ Sweden (e-mail: \{lindsten, schon\}@isy.liu.se) \\ ** Center of Mathematical Sciences, Lund University, Lund, Sweden \\ (e-mail: jimmy@maths.lth.se)
}

\begin{abstract}
Particle filters (PFs) have shown to be very potent tools for state estimation in nonlinear and/or non-Gaussian state-space models. For certain models, containing a conditionally tractable substructure (typically conditionally linear Gaussian or with finite support), it is possible to exploit this structure in order to obtain more accurate estimates. This has become known as Rao-Blackwellised particle filtering (RBPF). However, since the RBPF is typically more computationally demanding than the standard PF per particle, it is not always beneficial to resort to Rao-Blackwellisation. For the same computational effort, a standard PF with an increased number of particles, which would also increase the accuracy, could be used instead. In this paper, we have analysed the asymptotic variance of the RBPF and provide an explicit expression for the obtained variance reduction. This expression could be used to make an efficient discrimination of when to apply Rao-Blackwellisation, and when not to.
\end{abstract}

Keywords: Particle filtering, Monte-Carlo methods, Rao-Blackwellised particle filter, Marginalised particle filter, Rao-Blackwellisation, variance reduction

\section{INTRODUCTION AND RELATED WORK}

Many important problems in various fields of science are related to state estimation in general state-space models, based on noisy observations. If a prior distribution is assumed for the initial state, the optimal filter is given by the Bayesian filtering recursions. In a few special cases, basically for linear Gaussian state-space (LGSS) models and finite state-space (FSS) models, the optimal filtering problem is analytically tractable. However, many interesting problems do not exhibit such nice properties, but are both nonlinear and/or non-Gaussian. In these cases, the optimal filter needs to be approximated in some way. Sequential Monte Carlo methods, or particle filters (PFs), have shown to be very powerful tools when addressing such intractable models. Since the introduction of the PF by Gordon et al. (1993), we have experienced a vast amount of research in the area. For instance, many improvements and extensions have been introduced to increase the accuracy of the filter, see e.g. Doucet and Johansen (2010) for an overview of recent developments.

One natural idea is to exploit any tractable substructure in the model, see e.g. Doucet et al. (2000b); Schön et al. (2005). More precisely, if the model, conditioned on one partition of the state, behaves like e.g. an LGSS or an FSS it is sufficient to employ particles for the intractable part and make use of the analytic tractability for the remaining part. Inspired by the Rao-Blackwell theorem,

\footnotetext{
* This work was supported by the strategic research center MOVIII, funded by the Swedish Foundation for Strategic Research (SSF) and CADICS, a Linneaus Center funded by the Swedish Research Council.
}

this has become known as the Rao-Blackwellised particle filter (RBPF).

The motivation for the RBPF is to improve the accuracy of the filter, i.e. any estimator derived from the RBPF will intuitively have lower variance than the corresponding estimator derived from the standard PF. Informally, the reason for this is that in the $\mathrm{RBPF}$, the particles are spread in a lower dimensional space, yielding a denser particle representation of the underlying distribution. The improved accuracy is also something that is experienced by practitioners. However, it can be argued that it is still not beneficial to resort to Rao-Blackwellisation in all cases. The reason is that the RBPF is typically more computationally expensive per iteration, compared to the standard PF (e.g. for an RBPF targeting a conditional LGSS model, each particle is equipped with a Kalman filter, all which needs to be updated at each iteration). Hence, for a fixed computational effort, we can choose to either use Rao-Blackwellisation or to run a standard PF, but instead increase the number of particles. Both these alternatives will reduce the variance of the estimators. Hence, it is important to understand and to be able to quantify how large variance reduction we can expect from the RBPF, in order to make suitable design choices for any given problem.

In this paper we shall study the asymptotic (in the number of particles) variances for the RBPF and the standard $\mathrm{PF}$ (we shall throughout the paper use the abbreviation $\mathrm{SPF}$ when referring to the non-Rao-Blackwellised PF). We provide an explicit expression for the difference between the variance of an estimator derived from the SPF and the variance of the corresponding estimator derived from 
the RBPF. Doucet et al. (2000b) motivates the RBPF by concluding that the weight variance will be lower than for the SPF, but they do not consider the variances of any estimators. This is done by Chopin (2004), who, under certain assumptions, concludes that the variance of an estimator based on the SPF always is at least as high as for the RBPF. However, no explicit expression for the difference is given, and the test functions considered are restricted to one partition of the state-space. Doucet et al. (2000a) also analyse the RBPF and the reduction of asymptotic variance. However, they only consider an importance sampling setting and neglect the important resampling step. Karlsson et al. (2005) studies the problem empirically, by running simulations on a specific example. Here, they have also analysed the number of computations per iteration in the RBPF and SPF, respectively.

The rest of this paper is organised as follows. In Section 2 we present the PF framework and define the SPF and the RBPF. We thereafter introduce the natural estimators that can be derived from the two filters, and discuss their asymptotic properties, in Section 3. The main result is given in Section 4, where we provide an explicit expression for the difference in asymptotic variance for the two estimators. In Section 5 this expression is studied for a special case and in Section 6 we discuss how it can be used in the design of a PF. Finally, in Section 7 we draw conclusions.

\section{BACKGROUND}

\subsection{Notation}

All random variables are defined on a common probability space $(\Omega, \mathcal{F}, P)$. For a measurable space $(\mathrm{X}, \mathcal{X})$, we denote by $\mathbb{F}(\mathrm{X})$ the set of all $\mathcal{X}$-measurable functions from $\mathrm{X}$ to $\mathbb{R}$. For a measure $\mu$ on $\mathcal{X}$ and $f \in \mathbb{F}(\mathrm{X})$, satisfying $\int|f| d \mu<\infty$, we denote by $\mu(f)$ the integral $\int f d \mu$. For $p \geq 1, \mathrm{~L}^{p}(\mathrm{X}, \mu)$ denotes the set of functions $f \in \mathbb{F}(\mathrm{X})$ such that $\int|f|^{p} d \mu<\infty$.

Let $(\mathrm{X}, \mathcal{X})$ and $(\mathrm{Y}, \mathcal{Y})$ be two measurable spaces. A kernel $V$ from $\mathrm{X}$ to $\mathrm{Y}$ is a map $V:(\mathrm{X}, \mathcal{Y}) \rightarrow \mathbb{R}_{+}$, such that (i) for each $x \in \mathrm{X}$, the map $A \mapsto V(x, A)$ is a positive measure on $\mathcal{Y}$ (ii) for each $A \in \mathcal{Y}$, the map $x \mapsto V(x, A)$ is $\mathcal{X}$-measurable. A kernel is called a transition kernel if $V(x, \mathrm{Y})=1$ for any $x \in \mathrm{X}$. We shall sometimes write $V(A \mid x)$ instead of $V(x, A)$.

For two measures $\mu$ and $\nu$, we say that $\nu$ is absolutely continuous with respect to $\mu$ (written $\nu \ll \mu$ ) if $\mu(A)=$ $0 \Rightarrow \nu(A)=0$. By $\mathrm{N}\left(m, \sigma^{2}\right)$ we denote the Gaussian distribution with mean $m$ and variance $\sigma^{2}$. Finally, convergence in distribution and convergence in probability are denoted by $\stackrel{\mathrm{D}}{\longrightarrow}$ and $\stackrel{\mathrm{P}}{\longrightarrow}$, respectively.

\subsection{Particle filtering}

Let $\left\{X_{t}, t \in \mathbb{N}\right\}$ be a discrete time Markov process on the state-space $(X, \mathcal{X})$ (typically some power of the real line with the corresponding Borel $\sigma$-algebra). The process is hidden, but observed through the measurement sequence $\left\{Y_{t}, t \in \mathbb{N}\right\}$ defined on $(\mathrm{Y}, \mathcal{Y})$. For a given sequence of measurements $Y_{1: t}=\left\{Y_{1}, \ldots, Y_{t}\right\}$ (a similar notation shall be used for other sequences as well), the joint smoothing distribution is defined as

$$
\phi_{t}(A) \triangleq \mathrm{P}\left(X_{1: t} \in A \mid Y_{1: t}\right), \quad A \in \mathcal{X}^{t} .
$$

In particle filtering, we seek to approximate this distribution by a weighted particle system $\left\{x_{1: t}^{N, i}, w_{t}^{N, i}\right\}_{i=1}^{N}$. Observe that this is a triangular array of random variables and, though cumbersome, it is important to keep the dependence on $N$ in the notation. There are many different ways to generate such a particle system (see e.g. Doucet and Johansen (2010) for an overview), but common to all are the concepts of sequential importance sampling and resampling. Importance sampling is used to propagate a weighted sample, targeting the smoothing distribution at time $t-1$, into a weighted sample targeting the smoothing distribution at time $t$. This is done by sampling new particles from a proposal kernel (a transition kernel from $\mathrm{X}^{t-1}$ to $\left.\mathrm{X}^{t}\right)$

$$
R_{t-1}\left(\tilde{x}_{1: t-1}, d x_{1: t}\right) .
$$

The proposal kernel is chosen such that $\phi_{t} \ll \pi_{t}$, where we have defined the probability measure on $\mathcal{X}^{t}$,

$$
\pi_{t}\left(d x_{1: t}\right) \triangleq \int_{\mathrm{X}^{t-1}} \phi_{t-1}\left(d \tilde{x}_{1: t-1}\right) R_{t-1}\left(\tilde{x}_{1: t-1}, d x_{1: t}\right) .
$$

Besides from the absolute continuity condition, we shall not assume any restrictions on the choice of proposal kernel. For instance, it can depend on the measurement sequence, but we shall not make such dependence explicit. The distribution $\pi_{t}$ can be seen as the "proposed smoothing distribution" at time $t$ under the proposal kernel $R_{t-1}$.

To compensate for the fact that we sample from the wrong distribution, the samples are weighted. For this purpose we introduce the weight function

$$
W_{t-1}\left(x_{1: t}\right) \triangleq \frac{d \phi_{t}}{d \pi_{t}}\left(x_{1: t}\right)
$$

A well known problem in particle filtering is weight degeneracy, see e.g. Doucet et al. (2000b); Doucet and Johansen (2010). To remedy this the particle system can be resampled, i.e. particles with high weights are duplicated and particles with low weights are discarded. As previously mentioned, there are many options available to the user when designing a particle filter, e.g. in the choice of resampling scheme, if the number of particles shall be fixed or varying and when the resampling shall be performed. In this paper, the results will be given for the particle filter presented in Algorithm 1 below. Briefly, we consider arbitrary proposal kernels (2) (under the absolute continuity assumption) and multinomial resampling which is performed at each iteration of the algorithm. However, results similar to those presented in Section 4 could be obtained for other types of PFs as well, such as the auxiliary PF (Pitt and Shephard, 1999) and PFs with more sophisticated resampling schemes.

As previously mentioned, the PF presented in this section, i.e. which targets the "full" smoothing distribution (1), will throughout the remainder of this paper be denoted the standard particle filter (SPF).

\subsection{Rao-Blackwellised particle filter}

The idea underlying the Rao-Blackwellised particle filter is to exploit any tractable substructure in the targeted 


$$
\begin{gathered}
a_{N}^{2} \Omega_{N}^{-2} \sum_{i=1}^{N}\left(v^{N, i}\right)^{2} g\left(\chi^{N, i}\right) \stackrel{\mathrm{P}}{\longrightarrow} \gamma(g), \\
a_{N} \Omega_{N}^{-1} \max _{1 \leq i \leq N} v^{N, i} \stackrel{\mathrm{P}}{\longrightarrow} 0,
\end{gathered}
$$

as $N \rightarrow \infty$, for any $f \in \mathrm{A}$ and any $g \in \mathrm{W}$, where $\Omega_{N}=\sum_{j=1}^{N} v^{N, j}$.

In the following two theorems (slight modifications of what has previously been given by Douc and Moulines (2008)) we claim asymptotic normality for the weighted particle systems generated by the SPF and the RBPF, respectively. Theorem 1. (Asymptotic normality of the SPF) Assume that the initial particle system $\left\{x_{1}^{N, i}, w_{1}^{N, i}\right\}_{i=1}^{N}$ is asymptotically normal for $\left(\phi_{1}, \mathrm{~A}_{1}, \mathrm{~W}_{1}, \sigma_{1}, \phi_{1},\{\sqrt{N}\}\right)$. Define recursively the sets

$$
\begin{gathered}
\mathrm{A}_{t} \triangleq\left\{f \in \mathrm{L}^{2}\left(\mathrm{X}^{t}, \phi_{t}\right): R_{t-1}\left(\cdot, W_{t-1} f\right) \in \mathrm{A}_{t-1},\right. \\
\left.R_{t-1}\left(\cdot, W_{t-1}^{2} f^{2}\right) \in \mathrm{W}_{t-1}\right\}, \\
\mathrm{W}_{t} \triangleq\left\{f \in \mathrm{L}^{1}\left(\mathrm{X}^{t}, \phi_{t}\right): R_{t-1}\left(\cdot, W_{t-1}^{2}|f|\right) \in \mathrm{W}_{t-1}\right\} .
\end{gathered}
$$

Assume that, for any $t \geq 1, R_{t}\left(\cdot, W_{t}^{2}\right) \in \mathrm{W}_{t}$. Then, for any $t \geq 1$, the weighted particle system $\left\{x_{1: t}^{N, i}, w_{t}^{N, i}\right\}_{i=1}^{N}$ generated by the SPF is asymptotically normal for $\left(\phi_{t}, \mathrm{~A}_{t}, \mathrm{~W}_{t}, \sigma_{t}, \phi_{t},\{\sqrt{N}\}\right)$. The asymptotic variance is, for $f \in \mathrm{A}_{t}$, given by

$$
\begin{aligned}
\sigma_{t}^{2}(f) & =\sigma_{t-1}^{2}\left(R_{t-1}\left(\cdot, W_{t-1} \bar{f}\right)\right) \\
& \quad+\phi_{t-1}\left[R_{t-1}\left(\cdot,\left(W_{t-1} \bar{f}\right)^{2}\right)\right], \\
\bar{f} & =f-\phi_{t}(f) .
\end{aligned}
$$

Proof. See Appendix A.

Theorem 2. (Asymptotic normality of the RBPF) Under analogous conditions and definitions as in Theorem 1 , for any $t \geq 1$ the particle system $\left\{\xi_{1: t}^{N, i}, \omega_{t}^{N, i}\right\}_{i=1}^{N}$ generated by the RBPF is asymptotically normal for $\left(\phi_{t}^{m}, \mathrm{~A}_{t}^{m}, \mathrm{~W}_{t}^{m}, \tau_{t}, \phi_{t}^{m},\{\sqrt{N}\}\right)$. The asymptotic variance is, for $g \in A_{t}^{m}$, given by

$$
\begin{aligned}
\tau_{t}^{2}(g) & =\tau_{t-1}^{2}\left(R_{t-1}^{m}\left(\cdot, W_{t-1}^{m} \bar{g}\right)\right) \\
\quad+\phi_{t-1}^{m}\left[R_{t-1}^{m}\left(\cdot,\left(W_{t-1}^{m} \bar{g}\right)^{2}\right)\right], & \\
\bar{g} & =g-\phi_{t}^{m}(g) .
\end{aligned}
$$

Proof. See Appendix A.

Recall from Remark 2 that the SPF and the RBPF are really just two particle filters, targeting different distributions, hence the similarity between the two theorems above. Actually, we could have sufficed with one, more general, theorem applicable to both filters. The reason for why we have chosen to present them separately is for clarity and to introduce all the required notation.

As previously pointed out, the RBPF will intuitively produce better estimates than the SPF, i.e. we expect $\tau_{t}^{2}\left(\phi_{t}^{c}(f)\right) \leq \sigma_{t}^{2}(f)$. Let us therefore define the variance difference

$$
\Delta_{t}(f) \triangleq \sigma_{t}^{2}(f)-\tau_{t}^{2}\left(\phi_{t}^{c}(f)\right) .
$$

The problem that we are concerned with in this paper is to find an explicit expression for this quantity. This will be provided in the next section.

\section{THE MAIN RESULT}

To analyse the variance difference (14) we shall need the following assumption (similar to what is used by Chopin (2004)).

Assumption 1. For each $\tilde{\xi}_{1: t-1} \in \mathrm{X}_{\xi}^{t-1}$, the two measures

$$
\int_{\mathbf{X}_{z}^{t-1}} \phi_{t-1}^{c}\left(d \tilde{z}_{1: t-1} \mid \tilde{\xi}_{1: t-1}\right) R_{t-1}\left(\left\{\tilde{\xi}_{1: t-1}, \tilde{z}_{1: t-1}\right\}, d x_{1: t}\right)
$$

and

$$
a_{t}\left(\xi_{1: t}\right) R_{t-1}^{m}\left(\tilde{\xi}_{1: t-1}, d \xi_{1: t}\right) \pi_{t}^{c}\left(d z_{1: t} \mid \xi_{1: t}\right)
$$

agree on $\mathcal{X}^{t}$, for some positive function $a_{t}: \mathrm{X}_{\xi}^{t} \rightarrow \mathbb{R}$ and some transition kernel $\pi_{t}^{c}$ from $\mathrm{X}_{\xi}^{t}$ to $\mathrm{X}_{z}^{t}$, for which $\phi_{t}^{c}\left(\cdot \mid \xi_{1: t}\right) \ll \pi_{t}^{c}\left(\cdot \mid \xi_{1: t}\right)$.

The basic meaning of this assumption is to create a connection between the proposal kernels $R_{t-1}$ and $R_{t-1}^{m}$. It is natural that we need some kind of connection. Otherwise the asymptotic variance expressions (12) and (13) would be completely decoupled, and it would not be possible to draw any conclusions from a comparison. Still, as we shall see in the next section, Assumption 1 is fairly weak.

We are now ready to state the main result of this paper.

Theorem 3. Under Assumption 1, and using the definitions from Theorem 1 and Theorem 2 , for any $f \in \tilde{\mathrm{A}}_{t}$,

$$
\begin{aligned}
& \Delta_{t}(f)=\Delta_{t-1}\left(R_{t-1}\left(\cdot, W_{t-1} \bar{f}\right)\right) \\
& \quad+\phi_{t-1}^{m}\left[R_{t-1}^{m}\left(\cdot,\left(\frac{1-a_{t}}{a_{t}}\right)\left(W_{t-1}^{m} \bar{\psi}\right)^{2}+a_{t} \operatorname{Var}_{\pi_{t}^{c}}\left(W_{t-1} \bar{f}\right)\right)\right],
\end{aligned}
$$

where

$$
\begin{aligned}
\bar{\psi} & =\phi_{t}^{c}(f)-\phi_{t}(f), \\
\tilde{\mathrm{A}}_{t} & \triangleq\left\{f \in \mathbb{F}\left(\mathrm{X}^{t}\right): \phi_{t}^{c}(f) \in \mathrm{A}_{t}^{m}\right\} \cap \mathrm{A}_{t} .
\end{aligned}
$$

Proof. See Appendix A.

\section{RELATIONSHIP BETWEEN THE PROPOSALS KERNELS}

To understand the results given in the previous section, we shall have a closer look at the relationship between the proposal kernels induced by Assumption 1. We shall do this for a certain family of proposal kernels. More precisely, assume that the kernels can be written

$$
\begin{aligned}
& R_{t-1}\left(\tilde{x}_{1: t-1}, d x_{1: t}\right)=r_{t-1}\left(d x_{t} \mid x_{1: t-1}\right) \delta_{\tilde{x}_{1: t-1}}\left(d x_{1: t-1}\right) \\
& R_{t-1}^{m}\left(\tilde{\xi}_{1: t-1}, d \xi_{1: t}\right)=r_{t-1}^{m}\left(d \xi_{t} \mid \xi_{1: t-1}\right) \delta_{\tilde{\xi}_{1: t-1}}\left(d \xi_{1: t-1}\right)
\end{aligned}
$$

Informally, this means that when a trajectory $\left(x_{1: t}^{N, i}\right.$ or $\left.\xi_{1: t}^{N, i}\right)$ is sampled at time $t$, we keep the "old" trajectory up to time $t-1$ and simply append a sample from time $t$. This is the case for most PFs (when targeting the joint smoothing distribution), but not all, see e.g. the resamplemove algorithm by Gilks and Berzuini (2001).

Furthermore, let $r_{t-1}$ be factorised as

$$
\begin{aligned}
r_{t-1}\left(d x_{t} \mid\right. & \left.x_{1: t-1}\right) \\
& =q_{t-1}^{c}\left(d z_{t} \mid \xi_{1: t}, z_{1: t-1}\right) q_{t-1}^{m}\left(d \xi_{t} \mid \xi_{1: t-1}, z_{1: t-1}\right) .
\end{aligned}
$$


Assume that $q_{t-1}^{m} \ll r_{t-1}^{m}$ and define the kernel

$$
\begin{aligned}
& \nu_{t}\left(d z_{1: t} \mid \xi_{1: t}\right) \triangleq \frac{d q_{t-1}^{m}\left(\cdot \mid \xi_{1: t-1}, z_{1: t-1}\right)}{d r_{t-1}^{m}\left(\cdot \mid \xi_{1: t-1}\right)}\left(\xi_{t}\right) \\
& \quad \times \phi_{t-1}^{c}\left(d z_{1: t-1} \mid \xi_{1: t-1}\right) q_{t-1}^{c}\left(d z_{t} \mid \xi_{1: t}, z_{1: t-1}\right) .
\end{aligned}
$$

It can now be verified that the choice

$$
\begin{aligned}
a_{t}\left(\xi_{1: t}\right) & =\int_{\mathrm{X}_{z}^{t}} \nu_{t}\left(d z_{1: t} \mid \xi_{1: t}\right), \\
\pi_{t}^{c}\left(d z_{1: t} \mid \xi_{1: t}\right) & =\frac{\nu_{t}\left(d z_{1: t} \mid \xi_{1: t}\right)}{a_{t}\left(\xi_{1: t}\right)}
\end{aligned}
$$

satisfies Assumption 1, given that $\phi_{t}^{c}\left(\cdot \mid \xi_{1: t}\right) \ll \pi_{t}^{c}\left(\cdot \mid \xi_{1: t}\right)$.

Hence, the function $a_{t}$ takes the role of a normalisation of the kernel $\nu_{t}$ to obtain a transition kernel $\pi_{t}^{c}$. One interesting fact is that, from (17), we cannot guarantee that $\Delta_{t}(f)$ is nonnegative for arbitrary functions $a_{t}$. At first this might seem counterintuitive, since it would mean that the variance is higher for the RBPF than for the SPF. The explanation lies in that Assumption 1, relating the proposal kernels in the two filters, is fairly weak. In other words, we have not assumed that the proposal kernels are "equally good". For instance, say that the optimal proposal kernel is used in the SPF, whereas the RBPF uses a poor kernel. It is then no longer clear that the RBPF will outperform the SPF. In the next section we shall see that if both filters use their respective bootstrap proposal kernel, a case when the term "equally good" makes sense, then $\Delta_{t}(f)$ will indeed be nonnegative. However, for other proposal kernels, it is not clear that there is an analogue between the SPF and the RBPF in the same sense.

\subsection{Example: Bootstrap kernels}

Let $Q\left(d x_{t} \mid x_{t-1}\right)$ be the Markov transition kernel of the process $X$. In the bootstrap SPF we choose the proposal kernel according to (20) with

$$
r_{t-1}\left(d x_{t} \mid x_{1: t-1}\right)=Q\left(d x_{t} \mid x_{t-1}\right)
$$

where, almost surely, for $A \in \mathcal{X}$

$$
\begin{aligned}
Q\left(A \mid X_{t-1}\right) & =\mathrm{P}\left(X_{t} \in A \mid X_{t-1}\right) \\
& =\mathrm{P}\left(X_{t} \in A \mid X_{1: t-1}, Y_{1: t-1}\right) .
\end{aligned}
$$

The second equality follows from the Markov property of the process. In the RBPF, the analogue of the bootstrap proposal kernel is to use (21) with

$$
r_{t-1}^{m}\left(A \mid \Xi_{1: t-1}\right)=\mathrm{P}\left(\Xi_{t} \in A \mid \Xi_{1: t-1}, Y_{1: t-1}\right),
$$

for $A \in \mathcal{X}_{\xi}$.

It can be verified (see Appendix B) that these choices fulfill Assumption 1 with

$$
a_{t} \equiv 1
$$

and

$$
\pi_{t}^{c}\left(A \mid \Xi_{1: t}\right)=\mathrm{P}\left(Z_{1: t} \in A \mid \Xi_{1: t}, Y_{1: t-1}\right),
$$

for $A \in \mathcal{X}_{z}^{t}$. Hence, $\pi_{t}^{c}$ is indeed the predictive distribution of $Z_{1: t}$ conditioned on $\Xi_{1: t}$ and based on the measurements up to time $t-1$. In this case we can also write $\pi_{t}\left(d x_{1: t}\right)=$ $\pi_{t}^{m}\left(d \xi_{1: t}\right) \pi_{t}^{c}\left(d z_{1: t} \mid \xi_{1: t}\right)$, which highlights the connection between the predictive distributions in the two filters. In this case, due to (29), the variance difference (17) can be simplified to

$$
\begin{aligned}
\Delta_{t}(f)=\Delta_{t-1} & \left(R_{t-1}\left(\cdot, W_{t-1} \bar{f}\right)\right) \\
& +\phi_{t-1}^{m}\left[R_{t-1}^{m}\left(\cdot, \operatorname{Var}_{\pi_{t}^{c}}\left(W_{t-1} \bar{f}\right)\right)\right] .
\end{aligned}
$$

Hence, $\Delta_{t}(f)$ can be written as a sum (though, we have expressed it in a recursive form here) in which each term is an expectation of a conditional variance. It is thus ensured to be nonnegative.

\section{DISCUSSION}

In Theorem 3 we gave an explicit expression for the difference in asymptotic variance between the SPF and the RBPF. This expression can be used as a guideline for when it is beneficial to apply Rao-Blackwellisation, and when it is not. The variance expressions given in this paper are asymptotic. Consequently, they do not apply exactly to the variances of the estimators (6) and (7), for a finite number of particles. Still, a reasonable approximation of the accuracy of the estimator $(6)$ is

$$
\operatorname{Var}\left(\hat{f}_{\mathrm{SPF}}^{N}\right) \approx \frac{\sigma_{t}^{2}(f)}{N}
$$

and similarly for $(7)$

$$
\operatorname{Var}\left(\hat{f}_{\mathrm{RBPF}}^{N}\right) \approx \frac{\tau_{t}^{2}\left(\phi_{t}^{c}(f)\right)}{N} .
$$

Now, assume that the computational effort required by the RBPF, using $M$ particles, equals that required by the SPF, using $N$ particles (thus, $M<N$ since, in general, the RBPF is more computationally demanding than the SPF per particle). We then have

$$
\frac{\operatorname{Var}\left(\hat{f}_{\mathrm{SPF}}^{N}\right)}{\operatorname{Var}\left(\hat{f}_{\mathrm{RBPF}}^{M}\right)} \approx \frac{M}{N}\left(1+\frac{\Delta_{t}(f)}{\tau_{t}^{2}\left(\phi_{t}^{c}(f)\right)}\right) .
$$

Whether or not this quantity is greater than one tells us if it is beneficial to use Rao-Blackwellisation. The crucial point is then to compute the ratio $\Delta_{t}(f) / \tau_{t}^{2}\left(\phi_{t}^{c}(f)\right)$, which in itself is a challenging problem. We are currently investigating ways to estimate this ratio from a single run of the RBPF.

As a final remark, for the special case discussed in Section 5.1, the variance difference (31) resembles the last term in the expression (8). They are both composed of an expectation of a conditional variance. One important difference though, is that the dependence on the weight function $W_{t-1}$ is visible in (31). As an example, if the test function is restricted to $f \in \mathrm{L}^{1}\left(\mathrm{X}_{\xi}^{t}, \phi_{t}^{m}\right)$ the gain in variance indicated by (8) would be zero (since $\operatorname{Var}\left(f\left(\Xi_{1: t}\right) \mid \Xi_{1: t}\right) \equiv$ $0)$, but this is not the case for the actual gain (31).

\section{CONCLUSIONS}

We have analysed the Rao-Blackwellised particle filter in a fairly general setting, and provide an explicit expression for the reduction of asymptotic variance obtained from Rao-Blackwellisation. This expression is expected to be of practical use, since it can serve as an indicator for when it is beneficial to apply Rao-Blackwellisation, and when it is not. We are currently investigating efficient methods, based on the analytical expression, for estimating the obtained variance reduction.

\section{Appendix A. PROOFS}

Proof of Theorem 1. In Theorem 10 in Douc and Moulines (2008), take 


$$
L_{t-1}\left(\tilde{x}_{1: t-1}, d x_{1: t}\right)=W_{t-1}\left(x_{1: t}\right) R_{t-1}\left(\tilde{x}_{1: t-1}, d x_{1: t}\right)
$$

which satisfies the conditions of the hypothesis. Furthermore, $\phi_{t-1} L_{t-1}\left(\mathrm{X}^{t}\right)=1$. Now, the results follow for the choice $\kappa=0$.

In Theorem 10 in Douc and Moulines (2008), the asymptotic normality of a particle system obtained after resampling is considered. Compared to Theorem 1 of this paper, they thus obtain an additional term in the expression for the asymptotic variance.

Proof of Theorem 2. As the previous proof, with

$$
L_{t-1}^{m}\left(\tilde{\xi}_{1: t-1}, d \xi_{1: t}\right)=W_{t-1}^{m}\left(\xi_{1: t}\right) R_{t-1}^{m}\left(\tilde{\xi}_{1: t-1}, d \xi_{1: t}\right) .
$$

Proof of Theorem 3. Let Assumption 1 be satisfied. We shall start by determining the relationship between the weight functions $W_{t-1}$ and $W_{t-1}^{m}$. Consider

$$
\begin{aligned}
& \phi_{t}\left(d x_{1: t}\right)=\frac{d \phi_{t}}{d \pi_{t}}\left(x_{1: t}\right) \pi_{t}\left(d x_{1: t}\right) \\
& =W_{t-1}\left(x_{1: t}\right) \int_{\mathrm{X}^{t-1}} \phi_{t-1}\left(d \tilde{x}_{1: t-1}\right) R_{t-1}\left(\tilde{x}_{1: t-1}, d x_{1: t}\right)
\end{aligned}
$$

where we have made use of the definitions in (3) and (4). Furthermore, from the factorisation of $\phi_{t-1}$ (5) and Assumption 1 we get

$$
\begin{aligned}
& \phi_{t}\left(d x_{1: t}\right)=W_{t-1}\left(x_{1: t}\right) \int_{\mathrm{X}_{\xi}^{t-1}}\left(\phi_{t-1}^{m}\left(d \tilde{\xi}_{1: t-1}\right)\right. \\
& \left.\times \int_{\mathbf{X}_{z}^{t-1}} \phi_{t-1}^{c}\left(d \tilde{z}_{1: t-1} \mid \tilde{\xi}_{1: t-1}\right) R_{t-1}\left(\left\{\tilde{\xi}_{1: t-1}, \tilde{z}_{1: t-1}\right\}, d x_{1: t}\right)\right) \\
& =a_{t}\left(\xi_{1: t}\right) W_{t-1}\left(x_{1: t}\right) \\
& \times \int_{\mathbf{X}_{\xi}^{t-1}} \underbrace{\phi_{t-1}^{m}\left(d \tilde{\xi}_{1: t-1}\right) R_{t-1}^{m}\left(\tilde{\xi}_{1: t-1}, d \xi_{1: t}\right)}_{\text {integrates to } \pi_{t}^{m}\left(d \xi_{1: t}\right)} \pi_{t}^{c}\left(d z_{1: t} \mid \xi_{1: t}\right) \\
& =a_{t}\left(\xi_{1: t}\right) W_{t-1}\left(x_{1: t}\right) \pi_{t}^{m}\left(d \xi_{1: t}\right) \pi_{t}^{c}\left(d z_{1: t} \mid \xi_{1: t}\right),
\end{aligned}
$$

(recall that $\pi_{t}^{m}$ is defined analogously to $(3)$ ).

However, we may also write

$$
\phi_{t}=\frac{d \phi_{t}^{m}}{d \pi_{t}^{m}} \frac{d \phi_{t}^{c}}{d \pi_{t}^{c}} \pi_{t}^{m} \pi_{t}^{c}
$$

Hence, we have two candidates for the Radon-Nikodym derivative of $\phi_{t}$ with respect to $\pi_{t}^{m} \pi_{t}^{c}$ which, $\pi_{t}^{m} \pi_{t}^{c}$-almost surely, implies

$$
a_{t}\left(\xi_{1: t}\right) W_{t-1}\left(x_{1: t}\right)=W_{t-1}^{m}\left(\xi_{1: t}\right) \frac{d \phi_{t}^{c}\left(\cdot \mid \xi_{1: t}\right)}{d \pi_{t}^{c}\left(\cdot \mid \xi_{1: t}\right)}\left(z_{1: t}\right) .
$$

Now, consider an arbitrary $\varphi \in \tilde{\mathrm{A}}_{t}$. Using (5) and Assumption 1 we may write

$$
\phi_{t-1}\left[R_{t-1}(\cdot, \varphi)\right]=\phi_{t-1}^{m}\left[R_{t-1}^{m}\left(\cdot, a_{t} \pi_{t}^{c}(\varphi)\right)\right] .
$$

Comparing (A.6) and (12), we see that we can let $\varphi$ take the role of $\left(W_{t-1} \bar{f}\right)^{2}$. Hence, consider

$$
\pi_{t}^{c}\left(\left(W_{t-1} \bar{f}\right)^{2}\right)=\left(\pi_{t}^{c}\left(W_{t-1} \bar{f}\right)\right)^{2}+\operatorname{Var}_{\pi_{t}^{c}}\left(W_{t-1} \bar{f}\right),
$$

where, using (A.5) we have $\pi_{t}^{m}$-almost surely,

$$
\begin{aligned}
& \pi_{t}^{c}\left(W_{t-1} \bar{f}\right)= \int \frac{W_{t-1}^{m}\left(\xi_{1: t}\right)}{a_{t}\left(\xi_{1: t}\right)} \frac{d \phi_{t}^{c}\left(\cdot \mid \xi_{1: t}\right)}{d \pi_{t}^{c}\left(\cdot \mid \xi_{1: t}\right)}\left(z_{1: t}\right) \\
& \quad \times \bar{f}\left(\left\{\xi_{1: t}, z_{1: t}\right\}\right) \pi_{t}^{c}\left(d z_{1: t} \mid \xi_{1: t}\right) \\
&= \\
& \quad \frac{W_{t-1}^{m}\left(\xi_{1: t}\right)}{a_{t}\left(\xi_{1: t}\right)} \phi_{t}^{c}(\bar{f})=\frac{W_{t-1}^{m}\left(\xi_{1: t}\right)}{a_{t}\left(\xi_{1: t}\right)} \bar{\psi}\left(\xi_{1: t}\right) .
\end{aligned}
$$

Here we have made use of the definition of $\bar{\psi}$ in (18), yielding

$$
\phi_{t}^{c}(\bar{f})=\phi_{t}^{c}\left(f-\phi_{t}(f)\right)=\bar{\psi}\left(\xi_{1: t}\right) .
$$

Combining (A.7) and (A.8) we get, $\pi_{t}^{m}$-almost surely,

$$
\begin{aligned}
& a_{t}\left(\xi_{1: t}\right) \pi_{t}^{c}\left(\left(W_{t-1} \bar{f}\right)^{2}\right) \\
& \quad=\frac{\left(W_{t-1}^{m}\left(\xi_{1: t}\right) \bar{\psi}\left(\xi_{1: t}\right)\right)^{2}}{a_{t}\left(\xi_{1: t}\right)}+a_{t}\left(\xi_{1: t}\right) \operatorname{Var}_{\pi_{t}^{c}}\left(W_{t-1} \bar{f}\right) .
\end{aligned}
$$

Let $L_{t-1}$ and $L_{t-1}^{m}$ be defined as in (A.1) and (A.2), respectively. Then, $R_{t-1}\left(\cdot, W_{t-1} \bar{f}\right)=L_{t-1}(\cdot, \bar{f})$ and $R_{t-1}^{m}\left(\cdot, W_{t-1}^{m} \bar{\psi}\right)=L_{t-1}^{m}(\cdot, \bar{\psi})$.

Using (14), (12), (13) and the above results, the difference in asymptotic variance can be expressed as

$$
\begin{aligned}
& \Delta_{t}(f)=\sigma_{t-1}^{2}\left(L_{t-1}(\cdot, \bar{f})\right)-\tau_{t-1}^{2}\left(L_{t-1}^{m}(\cdot, \bar{\psi})\right) \\
& +\phi_{t-1}\left[R_{t-1}\left(\cdot,\left(W_{t-1} \bar{f}\right)^{2}\right)\right]-\phi_{t-1}^{m}\left[R_{t-1}^{m}\left(\cdot,\left(W_{t-1}^{m} \bar{\psi}\right)^{2}\right)\right] \\
& =\sigma_{t-1}^{2}\left(L_{t-1}(\cdot, \bar{f})\right)-\tau_{t-1}^{2}\left(L_{t-1}^{m}(\cdot, \bar{\psi})\right) \\
& +\phi_{t-1}^{m}\left[R_{t-1}^{m}\left(\cdot\left(\frac{1}{a_{t}}-1\right)\left(W_{t-1}^{m} \bar{\psi}\right)^{2}+a_{t} \operatorname{Var}_{\pi_{t}^{c}}\left(W_{t-1} \bar{f}\right)\right)\right]
\end{aligned}
$$

(recall that $\pi_{t}^{m}=\phi_{t-1}^{m} R_{t-1}^{m}$ which ensures that we, due to the expectation w.r.t. $\phi_{t-1}^{m} R_{t-1}^{m}$ in (A.11), can make use of the equality in (A.10)).

Finally, consider

$$
\begin{aligned}
& \phi_{t-1}^{c}\left(L_{t-1}(\cdot, \bar{f})\right)=\int_{\mathbf{X}^{t}}\left(W_{t-1}\left(x_{1: t}\right) \bar{f}\left(x_{1: t}\right)\right. \\
& \left.\times \int_{\mathbf{X}_{z}^{t-1}} \phi_{t-1}^{c}\left(d \tilde{z}_{1: t-1} \mid \tilde{\xi}_{1: t-1}\right) R_{t-1}\left(\left\{\tilde{\xi}_{1: t-1}, \tilde{z}_{1: t-1}\right\}, d x_{1: t}\right)\right) \\
& =\int_{\mathbf{X}_{\xi}^{t}} \int_{\mathbf{X}_{z}^{t}} a_{t}\left(\xi_{1: t}\right) W_{t-1}\left(\left\{\xi_{1: t}, z_{1: t}\right\}\right) \bar{f}\left(\left\{\xi_{1: t}, z_{1: t}\right\}\right) \\
& \quad \times R_{t-1}^{m}\left(\tilde{\xi}_{1: t-1}, d \xi_{1: t}\right) \pi_{t}^{c}\left(d z_{1: t} \mid \xi_{1: t}\right) \\
& =\int_{\mathbf{X}_{\xi}^{t}}\left(W_{t-1}^{m}\left(\xi_{1: t}\right) R_{t-1}^{m}\left(\tilde{\xi}_{1: t-1}, d \xi_{1: t}\right)\right. \\
& \left.\quad \times \int_{\mathbf{X}_{z}^{t}} \bar{f}\left(\left\{\xi_{1: t}, z_{1: t}\right\}\right) \frac{d \phi_{t}^{c}\left(\cdot \mid \xi_{1: t}\right)}{d \pi_{t}^{c}\left(\cdot \mid \xi_{1: t}\right)}\left(z_{1: t}\right) \pi_{t}^{c}\left(d z_{1: t} \mid \xi_{1: t}\right)\right) \\
& =L_{t-1}^{m}\left(\cdot, \phi_{t}^{c}(\bar{f})\right)=L_{t-1}^{m}(\cdot, \bar{\psi}), \quad \pi_{t}^{m} \text {-almost surely. }
\end{aligned}
$$

(the third equality follows from Assumption 1 and the fourth follows $\pi_{t}^{m} \pi_{t}^{c}$-almost surely from (A.5)).

Hence,

$$
\sigma_{t-1}^{2}\left(L_{t-1}(\cdot, \bar{f})\right)-\tau_{t-1}^{2}\left(L_{t-1}^{m}(\cdot, \bar{\psi})\right)=\Delta_{t-1}\left(L_{t-1}(\cdot, \bar{f})\right),
$$

which completes the proof. 


\section{Appendix B. VERIFICATION OF ASSUMPTION 1 FOR BOOTSTRAP KERNELS}

We shall now analyse Assumption 1 for the choice of proposal kernels used in Section 5.1. First, note that, for $A \in \mathcal{X}_{z}^{t}$,

$$
\phi_{t}^{c}\left(A \mid \Xi_{1: t}\right)=\mathrm{P}\left(Z_{1: t} \in A \mid \Xi_{1: t}, Y_{1: t}\right) .
$$

Now, let $\mu^{1}\left(\cdot \mid \tilde{\xi}_{1: t-1}\right)$ be the measure in (15). Let $\{A, B, C, D\}$ be a measurable rectangle, i.e. $A \in \mathcal{X}_{\xi}$, $B \in \mathcal{X}_{z}, C \in \mathcal{X}_{\xi}^{t-1}, D \in \mathcal{X}_{z}^{t-1}$, and consider

$$
\begin{aligned}
& \mu^{1}(A \times B\left.\times C \times D \mid \Xi_{1: t-1}\right) \\
&= \int_{A} \int_{B} \int_{C} \int_{D} \int_{\mathrm{X}_{z}^{t-1}} \phi_{t-1}^{c}\left(d \tilde{z}_{1: t-1} \mid \Xi_{1: t-1}\right) \\
& \times r_{t-1}\left(d\left\{\xi_{t}, z_{t}\right\} \mid\left\{\xi_{1: t-1}, z_{1: t-1}\right\}\right) \\
& \times \delta_{\Xi_{1: t-1}}\left(d \xi_{1: t-1}\right) \delta_{\tilde{z}_{1: t-1}}\left(d z_{1: t-1}\right) \\
&= \int_{A} \int_{B} \int_{C} \int_{D} \phi_{t-1}^{c}\left(d z_{1: t-1} \mid \Xi_{1: t-1}\right) \\
& \quad \times r_{t-1}\left(d\left\{\xi_{t}, z_{t}\right\} \mid\left\{\xi_{1: t-1}, z_{1: t-1}\right\}\right) \delta_{\Xi_{1: t-1}}\left(d \xi_{1: t-1}\right) \\
&=I_{C}\left(\Xi_{1: t-1}\right) \int_{A} \int_{B} \int_{D} \phi_{t-1}^{c}\left(d z_{1: t-1} \mid \Xi_{1: t-1}\right) \\
& \quad \times r_{t-1}\left(d\left\{\xi_{t}, z_{t}\right\} \mid\left\{\Xi_{1: t-1}, z_{1: t-1}\right\}\right) \\
&=I_{C}\left(\Xi_{1: t-1}\right) \mathrm{E}\left[\int_{A} \int_{B} r_{t-1}\left(d\left\{\xi_{t}, z_{t}\right\} \mid\left\{\Xi_{1: t-1}, Z_{1: t-1}\right\}\right)\right. \\
&\left.\quad \times I_{D}\left(Z_{1: t-1}\right) \mid \Xi_{1: t-1}, Y_{1: t-1}\right]
\end{aligned}
$$

/using (26) and (27)/

$$
\begin{aligned}
& =I_{C}\left(\Xi_{1: t-1}\right) \mathrm{E}\left[I_{D}\left(Z_{1: t-1}\right) \times\right. \\
& \left.\mathrm{E}\left[I_{A}\left(\Xi_{t}\right) I_{B}\left(Z_{t}\right) \mid \Xi_{1: t-1}, Z_{1: t-1}, Y_{1: t-1}\right] \mid \Xi_{1: t-1}, Y_{1: t-1}\right] \\
& =I_{C}\left(\Xi_{1: t-1}\right) \mathrm{E}\left[I_{D}\left(Z_{1: t-1}\right) I_{A}\left(\Xi_{t}\right) I_{B}\left(Z_{t}\right) \mid \Xi_{1: t-1}, Y_{1: t-1}\right]
\end{aligned}
$$

Similarly, let $\mu^{2}\left(\cdot \mid \tilde{\xi}_{1: t-1}\right)$ be the measure (16). We shall see that the two measures $\mu^{1}$ and $\mu^{2}$ agree if (29) and (30)) holds true. Consider

$$
\begin{aligned}
& \mu^{2}(A \times B \\
&= \times C \times D \mid \Xi_{A} \int_{B} \int_{C} \int_{D} \pi_{t}^{c}\left(d z_{1: t} \mid \xi_{1: t}\right) \\
& \times r_{t-1}^{m}\left(d \xi_{t} \mid \xi_{1: t-1}\right) \delta_{\Xi_{1: t-1}}\left(d \xi_{1: t-1}\right) \\
&= I_{C}\left(\Xi_{1: t-1}\right) \\
& \times \int_{A} \int_{B} \int_{D} \pi_{t}^{c}\left(d z_{1: t} \mid \Xi_{1: t-1}, \xi_{t}\right) r_{t-1}^{m}\left(d \xi_{t} \mid \Xi_{1: t-1}\right) \\
& / \operatorname{using}(28) / \\
&=I_{C}\left(\Xi_{1: t-1}\right) \\
& \quad \times \mathrm{E}\left[I_{A}\left(\Xi_{t}\right) \int_{B} \int_{D} \pi_{t}^{c}\left(d z_{1: t} \mid \Xi_{1: t}\right) \mid \Xi_{1: t-1}, Y_{1: t-1}\right]
\end{aligned}
$$

/integrating over $z_{1: t}$, using (30)/

$$
\begin{gathered}
=I_{C}\left(\Xi_{1: t-1}\right) \mathrm{E}\left[I_{A}\left(\Xi_{t}\right) \mathrm{E}\left[I_{B}\left(Z_{1: t-1}\right) I_{D}\left(Z_{t}\right) \mid \Xi_{1: t}, Y_{1: t-1}\right]\right. \\
\left.\qquad \mid \Xi_{1: t-1}, Y_{1: t-1}\right] \\
=I_{C}\left(\Xi_{1: t-1}\right) \mathrm{E}\left[I_{A}\left(\Xi_{t}\right) I_{B}\left(Z_{1: t-1}\right) I_{D}\left(Z_{t}\right) \mid \Xi_{1: t-1}, Y_{1: t-1}\right] .
\end{gathered}
$$

Hence, the two measures agree on measurable rectangles. Since the measurable rectangles form a $\pi$-system, generat- ing the product $\sigma$-algebra $\mathcal{X}^{t}=\mathcal{X}_{\xi} \times \mathcal{X}_{z} \times \mathcal{X}_{\xi}^{t-1} \times \mathcal{X}_{z}^{t-1}$, the two measures agree on $\mathcal{X}^{t}$ (Theorem 10.3 in Billingsley (1986)).

\section{REFERENCES}

Billingsley, P. (1986). Probability and measure. Wiley series in probability and mathematical statistics. John Wiley \& Sons, 2nd edition edition.

Chopin, N. (2004). Central limit theorem for sequential Monte Carlo methods and its application to Bayesian inference. The Annals of Statistics, 32(6), 2385-2411.

Douc, R. and Moulines, E. (2008). Limit theorems for weighted samples with applications to sequential Monte Carlo. The Annals of Statistics, 36(5), 2344-2376.

Doucet, A., de Freitas, N., Murphy, K., and Russell, S. (2000a). Rao-Blackwellised particle filtering for dynamic Bayesian networks. In Proceedings of the Sixteenth Conference on Uncertainty in Artificial Intelligence, 176-183. Stanford, USA.

Doucet, A., Godsill, S.J., and Andrieu, C. (2000b). On sequential Monte Carlo sampling methods for Bayesian filtering. Statistics and Computing, 10(3), 197-208.

Doucet, A. and Johansen, A. (2010). A tutorial on particle filtering and smoothing: Fifteen years later. In Handbook of Nonlinear Filtering (to appear). Oxford University Press.

Gilks, W.R. and Berzuini, C. (2001). Following a moving target - Monte Carlo inference for dynamic Bayesian models. Journal of the Royal Statistical Society. Series $B$ (Statistical Methodology), 63(1), 127-146.

Gordon, N.J., Salmond, D.J., and Smith, A.F.M. (1993). Novel approach to nonlinear/non-Gaussian Bayesian state estimation. Radar and Signal Processing, IEE Proceedings F, 140(2), $107-113$.

Karlsson, R., Schön, T.B., and Gustafsson, F. (2005). Complexity analysis of the marginalized particle filter. IEEE Transactions on Signal Processing, 53(11), 44084411.

Pitt, M.K. and Shephard, N. (1999). Filtering via simulation: Auxiliary particle filters. Journal of the American Statistical Association, 94(446), 590-599.

Schön, T.B., Gustafsson, F., and Nordlund, P.J. (2005). Marginalized particle filters for mixed linear/nonlinear state-space models. IEEE Transactions on Signal Processing, 53(7), 2279-2289.

Uglanov, A.V. (1991). Fubini's theorem for vector-valued measures. Math. USSR Sbornik, 69(2), 453-463. 